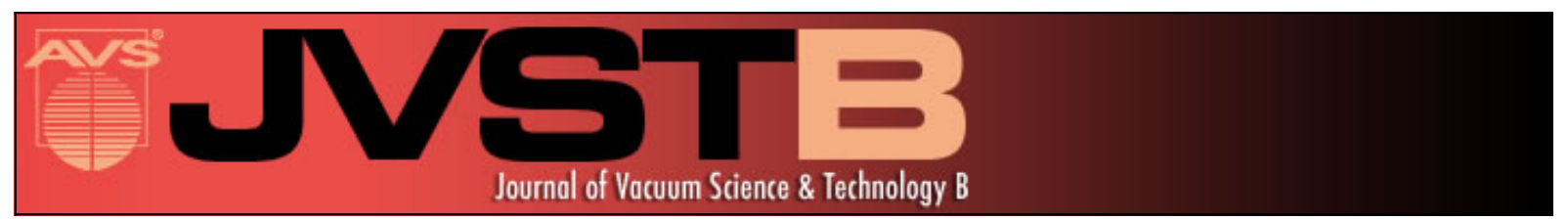

\title{
Crystallization of Ge in SiO2 matrix by femtosecond laser processing
}

Omer Salihoglu, Ula Kürüm, Halime Gul Yaglioglu, Ayhan Elmali, and Atilla Aydinli

Citation: Journal of Vacuum Science \& Technology B 30, 011807 (2012); doi: 10.1116/1.3677829

View online: http://dx.doi.org/10.1116/1.3677829

View Table of Contents: http://scitation.aip.org/content/avs/journal/jvstb/30/1?ver=pdfcov

Published by the AVS: Science \& Technology of Materials, Interfaces, and Processing

\section{Articles you may be interested in}

Structural and electronic characterization of $355 \mathrm{~nm}$ laser-crystallized silicon: Interplay of film thickness and laser fluence

J. Appl. Phys. 115, 163503 (2014); 10.1063/1.4872464

Surface fingerprints of individual silicon nanocrystals in laser-annealed Si/SiO2 superlattice: Evidence of nanoeruptions of laser-pressurized silicon

J. Appl. Phys. 111, 124302 (2012); 10.1063/1.4729303

Laser produced streams of Ge ions accelerated and optimized in the electric fields for implantation into $\mathrm{SiO} 2$ substratesa)

Rev. Sci. Instrum. 83, 02B305 (2012); 10.1063/1.3660819

Femtosecond laser crystallization of amorphous Ge

J. Appl. Phys. 109, 123108 (2011); 10.1063/1.3601356

In situ micro Raman investigation of the laser crystallization in Si thin films plasma enhanced chemical vapor deposition-grown from He-diluted $\mathrm{SiH} 4$

J. Appl. Phys. 95, 5366 (2004); 10.1063/1.1699506

\section{HDDEN}

\section{Instruments for Advanced Science}

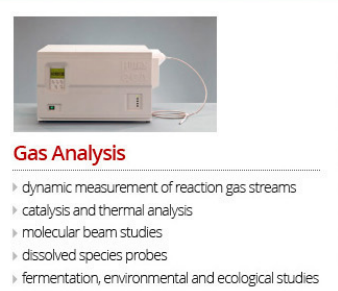

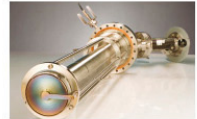

Plasma Diagnostics plasma source characterization etch and deposition process reaction

SIMS end point detection in ion beam etch
, analysis of neytrat and radicat species

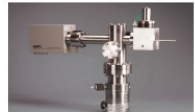

Vacuum Analysis partial pressure measurement and contro of process gases reactive sputter process control vacuum diagnostics vacuum coating process monitoring 


\title{
Crystallization of $\mathrm{Ge}$ in $\mathrm{SiO}_{2}$ matrix by femtosecond laser processing
}

\author{
Omer Salihoglua) \\ Advanced Research Laboratories, Bilkent University, Ankara 06800, Turkey \\ Ulaş Kürüm, Halime Gul Yaglioglu, and Ayhan Elmali \\ Department of Engineering Physics, Ankara University, 06100 Ankara, Turkey \\ Atilla Aydinli \\ Physics Department, Bilkent University, Ankara 06800, Turkey
}

(Received 28 October 2011; accepted 28 December 2011; published 19 January 2012)

\begin{abstract}
Germanium nanocrystals embedded in a siliconoxide matrix has been fabricated by single femtosecond laser pulse irradiation of germanium doped $\mathrm{SiO}_{2}$ thin films deposited with plasma enhanced chemical vapor deposition. SEM and AFM are used to analyze surface modification induced by laser irradiation. Crystallization of Ge in the oxide matrix is monitored with the optic phonon at $300 \mathrm{~cm}^{-1}$ as a function of laser fluence. Both the position the linewidth of the phonon provides clear signature for crystallization of Ge. In PL experiments, strong luminescence around $600 \mathrm{~nm}$ has been observed. (C) 2012 American Vacuum Society. [DOI: 10.1116/1.3677829]
\end{abstract}

\section{INTRODUCTION}

Various nanocrystals both metallic and semiconductor including $\mathrm{Ge}$ embedded in $\mathrm{SiO}_{2}$ matrix have recently received great attention due to their prospective applications in nanoelectronics $^{1,2}$ and optoelectronics ${ }^{3-5}$ especially in nonvolatile memory devices. ${ }^{6-8}$ Photoluminescence, due both to defects as well as to quantum confined carriers in Ge nanocrystals have been observed. ${ }^{9-11}$ Ge nanocrystals embedded in siliconoxide are being considered for nanostructure based solar cells due to their relatively large absorption coefficient and convenient gap (lower than that of Si QDs) and the ability to modulate the and gap with size. Various approaches for fabrication of matrix based nanocrystals have been used, including ion implantation, ${ }^{12}$ magnetron sputtering ${ }^{13}$ and plasma enhanced thin film deposition and as well as oxidation of polycrystalline layers as in the case of SiGe. ${ }^{14}$ Crystallization is typically induced by long term annealing in a high temperature furnace which is costly.

In cases where the sample may not be subjected to high temperatures for long periods, both $\mathrm{cw}$ and pulsed laser annealing may also be used. Many types of pulsed lasers have been used to anneal solids in the past, including those with nano-, ${ }^{15}$ pico- $^{16}$ and femtosecond ${ }^{17,18}$ pulse durations. Among them femtosecond pulses can interact with the material faster than the time required for electron phonon interaction and can cause ultrafast melting of the material ${ }^{19}$ during which negligible lateral heat diffusion takes place. This is in contrast with long laser pulses where rate of energy deposition and rate of three dimensional energy dissipation into the material are comparable for homogenous materials. In the case of crystalline semiconductors, it has been shown that femtosecond optical pulses can excite significant numbers of the valance electrons in the semiconductor. The photoexcited electrons then weaken the lattice and lead to structural transformation leading to nonthermal melting since the elec-

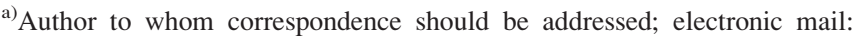
omersalihoglu@yahoo.com
}

tronic system and lattice are not in thermal equilibrium in the subpicosecond time scale. ${ }^{20}$ In wide band gap materials, multiphoton absorption is the dominant mechanism for deposition of the laser energy.

Fabrication of Ge nanocrystals in $\mathrm{SiO}_{2}$ matrix by femtosecond annealing is very promising since it is compatible with CMOS processes. Unlike thermal annealing, pulse laser annealing (PLA) provides local modification of surfaces down to diffraction limited spot sizes with low thermal budget and faster processing times when compared with high temperature furnace annealing. Furthermore, due to its surface initiated mechanism, femtosecond laser crystallization relatively cheap substrates such as glass or plastic may be used to deposit thin films. Annealing via femtosecond laser pulses also provide some useful advantages like chemical cleanliness, controlled thermal penetration and modification speed.

\section{EXPERIMENT}

Amorphous $\mathrm{SiO}_{2}$ films containing germanium $(\mathrm{Ge})$ was prepared by plasma enhanced chemical vapor deposition (PECVD). Full 3" (100) double side polished silicon wafers were used. Growth of a-Ge film was carried out in PlasmaLab $8510 \mathrm{C}$ reactor at $350{ }^{\circ} \mathrm{C}$ and the process was carried out under the pressure of 1 Torr and RF power of $12 \mathrm{~W}$. Flow rates were $200 \mathrm{sccm}$ for $\mathrm{GeH}_{4}(\% 2 \mathrm{in}$. He), $180 \mathrm{sccm}$ for $\mathrm{SiH}_{4}(\% 2$ in. $\mathrm{He})$ and $45 \mathrm{sccm}$ for $\mathrm{N}_{2} \mathrm{O}$. Final thickness of the germanium layer was about $600 \mathrm{~nm}$. Optical absorption of the film was measured as $\% 18$ at $800 \mathrm{~nm}$. Samples were cleaved into $1 \mathrm{~cm}$ by $1 \mathrm{~cm}$ pieces for different laser irradiation sequences.

A Ti:sapphire femtosecond pulsed laser-amplifier system (Spectra Physics Tsunami-Spitfire Pro XP) with $1 \mathrm{kHz}$ repetition rate and $800 \mathrm{~nm}$ wavelength was used for the experiments. The energy per pulse for the experiments was adjusted to $30 \mu \mathrm{J}$. Pulse duration of the system was changed to $40,80,120$ and 160 femtoseconds via compressor delay in the amplifier. A three-axis computer controlled motorized 
translational stage was used to illuminate different positions on the samples as well as to alter the fluences at the illuminated spots. The sample was mounted on the motorized stage and placed at the focal point of the laser [Fig. 1(c)]. Fluence in $\mathrm{mJ} / \mathrm{cm}^{2}$ was adjusted by moving the sample away from the focal point of the lens paying attention to obtaining homogenous beam spots. Translational stage was programmed such that after each line scan, motorized stage moves one step away from the optimum focal point along the optical axis to change the spot size of the focused laser beam and one step vertically to start a new line scan on fresh a-Ge surface [Fig. 1(a)]. This method creates irradiated spots with different fluences for each line on the sample. The spot sizes of the laser at position of the sample were measured by using a razor blade technique with an accuracy of $\pm 5 \mu \mathrm{m} .{ }^{21}$ Minimum laser spot size on the sample was $30 \mu \mathrm{m}$. We check the beam profile with a beam spot analyzer, Newport LBP-4, before and after the focal point of the laser beam and find it to be Gaussian [Fig. 1(b)]. The overall error in the measured energy density is less than $1 \%$. A commercial scanner (Newport Model:M-IMS300LM) was used with horizontal scan speed of $400 \mathrm{~mm} / \mathrm{s}$. Movement of the translational stage created 30 different irradiation lines containing separate laser treated spots in amorphous germanium which were irradiated with fluences ranging from $18 \mathrm{~mJ} / \mathrm{cm}^{2}$ to $2000 \mathrm{~mJ} / \mathrm{cm}^{2}$. Each line contains 25 identical laser spots which are irradiated with same fluence of the laser with center to center separation of $400 \mu \mathrm{m}$ [Fig. 1(a)]. Although only one spot is enough for measurements, many spots give us a chance to check homogeneity of the laser beam from one spot to another. All laser treatments have been done under ambient temperature and pressure. Laser irradiated samples were analyzed with optical microscope, scanning electron microscopy (SEM), Raman scattering and x-ray photoelectron spectroscopy (XPS). Composition of the film has been determined as $\mathrm{Si}_{0.2} \mathrm{O}_{0.5} \mathrm{Ge}_{0.3}$ by using XPS via sputtering.

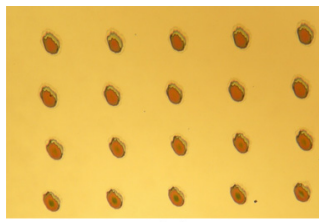

(a)

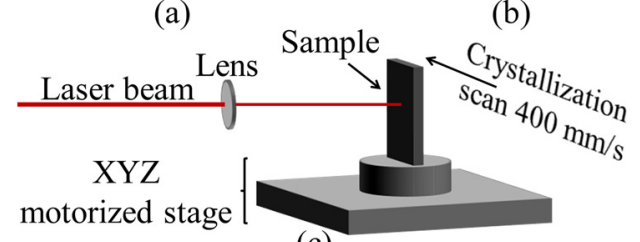

(c)

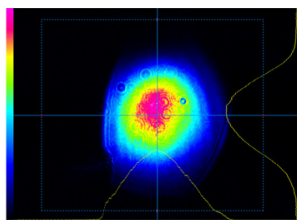

(b) tal setup. (a) Optical microscope image of FIG. 1. (Color online) Experimental setup. (a) Optical microscope image of
the laser treated $\mathrm{Si}_{0.2} \mathrm{O}_{0.5} \mathrm{Ge}_{0.3}$ surface. Each line contains identical laser marks on the surface. (b) Beam profile of the femtosecond laser beam near the focal point of the laser beam. (c) Simplified schematic of the laser treatment system. A linear stage was used to reach high speed enough $(400 \mathrm{~mm} / \mathrm{s})$ to get well separated single laser marks on the surface.

\section{RESULTS AND DISCUSSION}

The total amount of laser light absorbed during the pulse as well as the rate at which it is absorbed is critical for the modification of the material under irradiation. When $800 \mathrm{~nm}$ laser beam passes through a large bandgap material such as silicon dioxide, only a negligible fraction of beam energy is normally absorbed in the glass sample. $\mathrm{SiO}_{2}$ has a band gap of about $9 \mathrm{eV}$, much larger than the $1.55 \mathrm{eV}$ photons, the laser beam can deliver for band to band absorption. However, in the case of high intensity femtosecond lasers, nonlinear multiphoton absorption is the dominant mechanism for initiating the process of photoionization in glasses. ${ }^{21}$ This mechanism is responsible for heating, melting and ablation as well as swelling in the $\mathrm{SiO}_{2}$ :Ge sample. In our case, band to band absorption due to germanium may also contribute depending on Ge concentration in the oxide matrix. Clarifying the details of the absorption mechanism is beyond the scope of this paper. Here, we concentrate on the effects of the femtosecond beam on the material itself. Therefore, samples were characterized upon irradiation, samples with various above mentioned analytical tools.

First, a surface profilometer (Sloan Dektak 3030ST) was used to characterize topography of the irradiated spots. Figure 2 shows a line scan analysis of two samples irradiated at 97 and $115 \mathrm{~mJ} / \mathrm{cm}^{2}$ and summary of the line scan analysis for all fluences. From the data it is clear that the laser beam causes swelling at the surface and the amount of swelling increases with increasing fluence. The formation of swelling suggests that a liquid phase is locally formed by femtosecond laser irradiation ${ }^{23}$ accompanied with microexplosions in

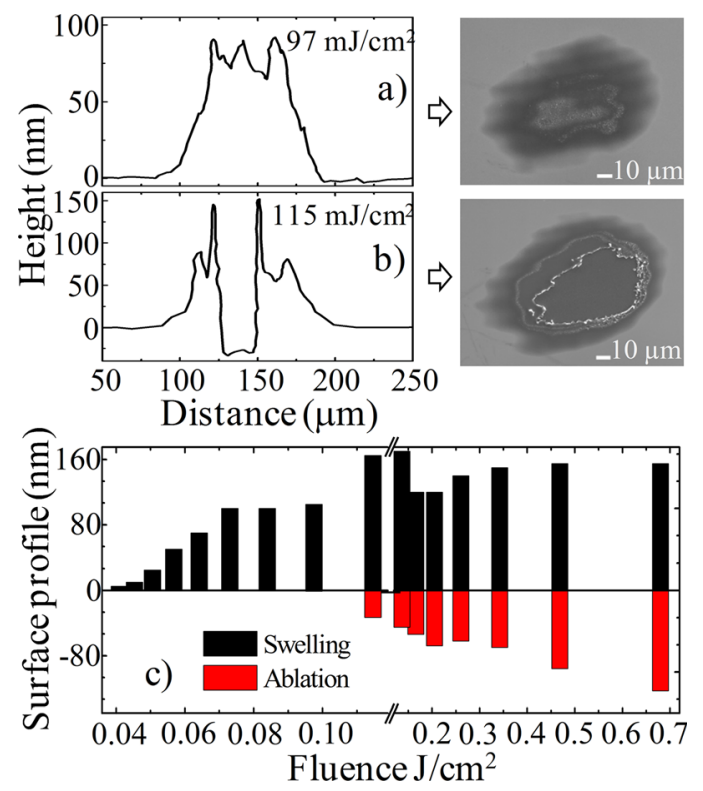

FIG. 2. (Color online) Surface profile analysis. Sloan Dektak 3030ST surface profilometer was used to measure surface profile. Swelling is observed for fluences higher than $40 \mathrm{~mJ} / \mathrm{cm}^{2}$. (a) surface profile of the spot irradiated with $100 \mathrm{~mJ} / \mathrm{cm}^{2}$, (b) surface profile of the spot irradiated with $115 \mathrm{~mJ} / \mathrm{cm}^{2}$ (ablation onset). (c) Summary of all surface profile results for the all fluences. Black bars represent swelling and red bars represent ablation. Although similar results were obtained from all pulse durations used in this experiment, data from 120 fs pulses was used in this graph. 
the film pushing up the molten $\mathrm{SiO}_{2}$ causing swelling of the material. In our samples, we observe that swelling starts at $40 \mathrm{~mJ} / \mathrm{cm}^{2}$ laser fluence. As the laser fluence increases swelling also increases. For laser fluences larger than $115 \mathrm{~mJ} / \mathrm{cm}^{2}$, ablation was observed. Ablation starts when microexplosions reach to some critical level such that the material inside the film is pushed completely out of the film where the laser spot is hot. ${ }^{24}$ Figures 2(a) and 2(b) show SEM images of the irradiated surface with the corresponding surface profile scans measured near the ablation threshold. Figure 2(a) shows the SEM image and corresponding surface profile of the irradiated spot that swells but ablation is not yet initiated. Figure 2(b) shows SEM image and line scan profile of the irradiated surface profile of an irradiated spot After the onset of ablation. When the laser fluence reaches to around $115 \mathrm{~mJ} / \mathrm{cm}^{2}$, ablation starts culminating in a crater like structure. Energy transport in the ablation process can be divided into two stages: the photon energy absorption, mainly through free electron generation and heating, and the redistribution of the absorbed energy to the lattice, leading to material removal. ${ }^{25}$ While SEM image in Fig. 2(b) clearly shows ablation, SEM image in Fig. 2(a) shows swelling without ablation. A summary of the line scan analysis of all irradiated spots is given in Fig. 2(c). Black bars represent swelling and red bars represent ablation. Figure 2(c) indicates that swelling occurs fluences higher than $40 \mathrm{~mJ} / \mathrm{cm}^{2}$ and ablation starts beyond fluences of $115 \mathrm{~mJ} / \mathrm{cm}^{2}$. Although similar results were obtained for all pulse durations used in this experiment, data from 120 fs pulses was used in Fig. 2. SEM images show an interference effect which we identified as laser-induced periodic surface structure (LIPSS). ${ }^{26}$ Periodic structures arise from the interference between incident laser light and the scattered or diffracted light parallel to the surface. As LIPPS is not the focus of this work and does not effect our Raman measurements, we will not discuss these structures in this manuscript further.

Raman spectroscopy provides one of the best ways of probing crystallization of the laser modified surface. Under proper conditions, it also gives clues about the crystal size and stress associated with the crystals. We have used Horiba LabRAM HR microRaman spectrometer to observe the Raman signal using $532.1 \mathrm{~nm}$ line of a doubled Nd:YAG laser, with a spot size of $4-5 \mu \mathrm{m}$. Figure 3 shows Raman spectra for samples irradiated with 120 fs laser pulses with different fluences. For fluences less than $45 \mathrm{~mJ} / \mathrm{cm}^{2}$ there is no sign of laser crystallization of Ge. Only a broad band representative of the local density of states for amorphous germanium Raman signal was observed for both as deposited and irradiated samples indicating that laser fluence in this range was not enough to create crystallized material. At the fluence of $45 \mathrm{~mJ} / \mathrm{cm}^{2}$ a sharp peak superimposed on the original broad peak appears around $298 \mathrm{~cm}^{-1}$, Raman intensity increasing with increasing irradiation laser fluence between $45 \mathrm{~mJ} / \mathrm{cm}^{2}$ to $65 \mathrm{~mJ} / \mathrm{cm}^{2}$, decreases very slowly between 64 $\mathrm{mJ} / \mathrm{cm}^{2}$ and $340 \mathrm{~mJ} / \mathrm{cm}^{2}$ and vanishes after $680 \mathrm{~mJ} / \mathrm{cm}^{2}$. This Vanishing may be related to defective crystalline material left behind after ablation. ${ }^{27}$ Not surprisingly silicon Raman line around $520 \mathrm{~cm}^{-1}$ appear for the fluences higher

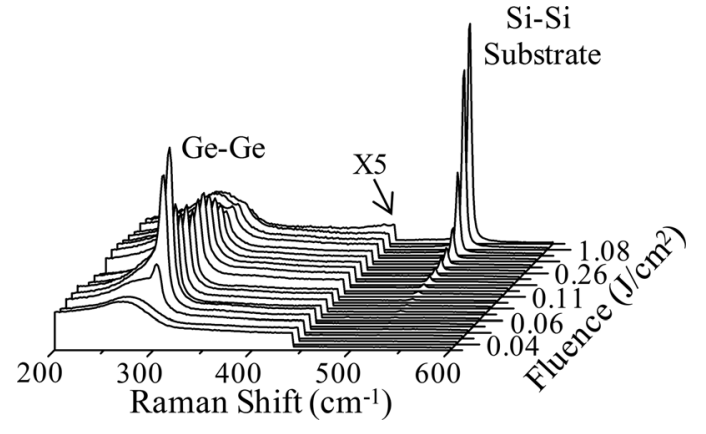

FIG. 3. Raman spectra of the $\mathrm{Si}_{0.2} \mathrm{O}_{0.5} \mathrm{Ge}_{0.3}$ as a function of single shot laser fluence. Laser at $800 \mathrm{~nm}$ with $120 \mathrm{fs}$ pulsewidth was used to irradiate the Germanium rich silicon dioxide film. Ge-Ge optic phonon line visible around $298 \mathrm{~cm}^{-1}$ and $\mathrm{Si}-\mathrm{Si}$ optic phonon line visible around $520 \mathrm{~cm}^{-1}$. Laser fluence for best crystallization with the narrowest Raman line occurred at the laser fluence of $85 \mathrm{~mJ} / \mathrm{cm}^{2}$.

than $75 \mathrm{~mJ} / \mathrm{cm}^{2}$. We believe that Raman signal for the silicon peak comes from the Si substrate. Si Raman peak intensity increases with fluence of the laser showing ablation of the $\mathrm{Si}_{0.2} \mathrm{O}_{0.5} \mathrm{Ge}_{0.3}$ layer. Ablation allows more laser beam to reach the substrate and leading to larger Si Raman signal. Raman Shift of the Si-Si optical phonon line stays constant around $521 \mathrm{~cm}^{-1}$ and Raman shift of Ge-Ge optical phonon line appear around $298 \mathrm{~cm}^{-1}$ with $\pm 0.3 \mathrm{~cm}^{-1}$ variations. We see that silicon signal is always at the same location verifying that it comes from the substrate. Ge signal varying very slowly indicating that Germanium crystals are subjected to same amount of stress inside the silicon oxide film. Raman linewidt is measured as $9.5 \mathrm{~cm}^{-1}$ at $55 \mathrm{~mJ} / \mathrm{cm}^{2}$ and it is narrowing down to $7.6 \mathrm{~cm}^{-1}$ at $85 \mathrm{~mJ} / \mathrm{cm}^{2}$ to give lowest Ge-Ge optical phonon linewidth then it increase with increasing fluence after $85 \mathrm{~mJ} / \mathrm{cm}^{2}$. Raman linewidth gives information about crystal size distribution and crystal quality therefore $85 \mathrm{~mJ} / \mathrm{cm}^{2}$ can be identified as the optimum crystallization fluence for our experiment.

Analysis of the Raman signal has been done for each sample to get values for linewidth, frequency shift and intensity of the Raman peaks. Width of the Raman signal gives information about crystal quality, shift of the Raman signal gives information about stress and intensity of the Raman signal gives information about density of the nano crystals. As deposited $\mathrm{SiO}_{2}$ :Ge films have a broad Raman signal around $270 \mathrm{~cm}^{-1}$ which has linewidth of $\sim 60 \mathrm{~cm}^{-1}$ suggestive of density of phonon states for a-Ge [Fig. 4(a)]. Similar observations have been reported by others with a broad Raman line centered at $270 \mathrm{~cm}^{-1} \cdot{ }^{28,29}$ As the fluence of the laser irradiation is increased we observe a peak at $297 \mathrm{~cm}^{-1}$ becomes pronounced superimposed on this broad signal indicating that crystallization of Ge has taken place. The intensity and linewidth of the Ge-Ge optic phonon line around $300 \mathrm{~cm}^{-1}$ change with irradiation laser fluence. A sample of the deconvolution applied to experimental data is shown in Fig. 4(b). Raman spectra contains two peaks around $270 \mathrm{~cm}^{-1}$ and $300 \mathrm{~cm}^{-1}$ corresponding to amorphous germanium and crystallized germanium optic phonon lines, respectively. Two Voight functions have been used to fit each Raman data [Fig. 4(b)]. Figure 4(c) shows the summary of 

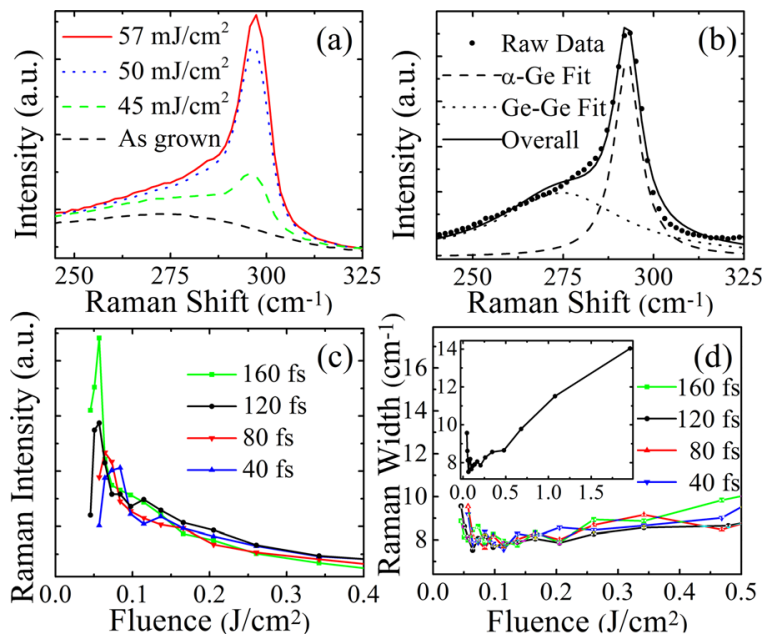

FIG. 4. (Color online) Ge-Ge optic phonon line Raman data. (a) Fluence vs Raman intensity, Ge optic phonon line starts to appear around $300 \mathrm{~cm}^{-1}$ at $45 \mathrm{~mJ} / \mathrm{cm}^{2}$ and its intensity increases and linewidth decreases with increasing laser fluence. (b) Deconvolution of the experimental Raman spectrum. (c) Raman intensity vs laser fluence. Around the $55 \mathrm{~mJ} / \mathrm{cm}^{2}$ higher pulse duration gives higher Raman intensity. (d) Linewidth of the Ge optic phonon at $300 \mathrm{~cm}^{-1}$ as a function of laser fluence provides a signature for the crystallization of Ge. Inset shows linewidth of the sample treated with $120 \mathrm{fs}$ pulse duration.

the Raman intensity of the Ge optic phonon line versus fluence for 40, 80, 120 and 160 fs pulse durations. After brief sharp increase around $55 \mathrm{~mJ} / \mathrm{cm}^{2}$, Raman intensity decreases with increasing fluence. Around $55 \mathrm{~mJ} / \mathrm{cm}^{2}$, high Raman intensity may be due to denser crystallization for longer pulse durations. Linewidth of the Ge optic phonon at $300 \mathrm{~cm}^{-1}$ as a function of laser fluence provides a signature for the crystallization of Ge [Fig. 4(d)]. Linewidth of Ge optic phonon is $2.36 \mathrm{~cm}^{-1}$ for single crystal $\mathrm{Ge} .^{30}$ Except shallow drop to $7.6 \mathrm{~cm}^{-1}$ around $85 \mathrm{~mJ} / \mathrm{cm}^{2}$, We measure an almost constant value of $8 \mathrm{~cm}^{-1}$ for samples irradiated between $45 \mathrm{~mJ} / \mathrm{cm}^{2}$ and $400 \mathrm{~m} \mathrm{~J} / \mathrm{cm}^{2}$. It then increases with increasing laser fluence [inset of Fig. 4(d)]. For the highest fluences (greater than $700 \mathrm{~mJ} / \mathrm{cm}^{2}$ ) narrow crystalline Ge phonon line at $300 \mathrm{~cm}^{-1}$ disappear and only a very broad amorphous germanium peak re-appear on the spectra. This is most likely due to formation of very small crystallites arising from ultrafast solidification. We have repeated this experiment many times for different pulse durations of $40 \mathrm{fs}, 80 \mathrm{fs}, 120 \mathrm{fs}$, and 160 fs and did not measure observable differences in the Raman linewidth when the pulse durations were varied.

The linewidth and shift of the Raman frequency depends on several factors such as crystallite size and defects, finite size effects ${ }^{31,32}$ as well as stress. It is well known that tensile stress results in Stokes frequency to red shift, while compressive stress leads to blue shift. ${ }^{31}$ In semiconductors such as $\mathrm{Si}$ and Ge, Stokes linewidth and frequency shift towards shorter wavenumbers when the crystal sizes become smaller than $5 \mathrm{~nm} .{ }^{32}$ Since it is well known that finite size effects cause larger Raman line widths, increase in the Raman linewidth beyond laser fluence of $100 \mathrm{~mJ} / \mathrm{cm}^{2}$ may be due to the formation of smaller nanocrystals upon ultrafast solidification. ${ }^{32}$ Alternatively, high defect densities in the crystallized

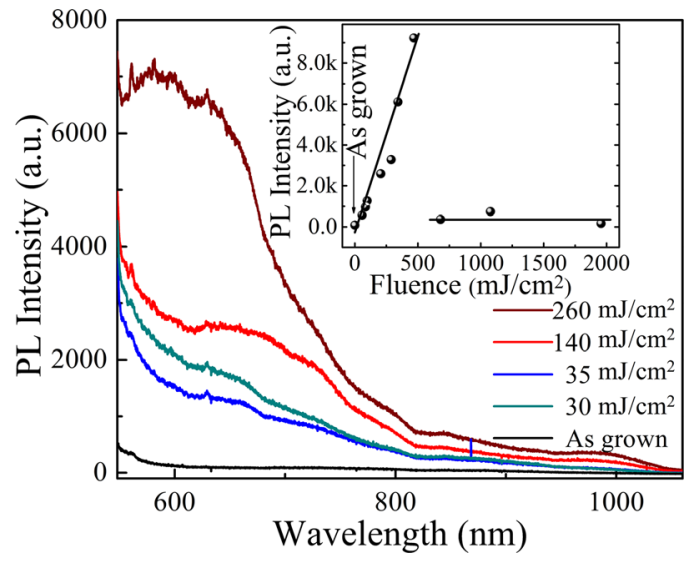

FIG. 5. (Color online) PL intensity vs wavelength for selected laser irradiated spots. PL intensity increases with increasing irradiating laser fluence up to the laser fluence of $470 \mathrm{~mJ} / \mathrm{cm}^{2}$. inset shows maximum PL intensity vs fluence graph. Although similar results were obtained from all pulse durations used in this experiment, data from 120 fs pulses was used in this graph.

Ge may also cause broader linewidth in the Raman spectra. ${ }^{33}$ TEM study on femtosecond laser ablation of amorphous silicon by Rogers et al. showed that ablation leaves behind defective crystalline material. $^{27}$

We have also measured photoluminescence from the irradiated samples using $532 \mathrm{~nm}$ line of the doubled YAG laser as the excitation source. We find that as-grown samples do not display PL signal. However, laser irradiated samples show strong luminescence in the visible region $(\sim 600 \mathrm{~nm})$. Figure 5 shows PL spectra for selected laser irradiated spots which are irradiated with pulses of 120 fs duration. PL intensity increases with increasing irradiating laser fluence up to the laser fluence of $470 \mathrm{~mJ} / \mathrm{cm}^{2}$. It then drops sharply to the level of luminescence from as-grown samples (Fig. 5, inset). Each broad PL spectrum is composed of multiple overlapping peaks. Similar PL signal have been observed by others. $^{34-38}$ Defect centers in the visible luminescence of $\mathrm{SiO}_{2}$ :Ge film cause this visible luminescence. ${ }^{28,36}$ Drop in PL signal at high fluencies (Fig. 5(a), inset) may be due to high fluences giving rise to different defect centers, suppressing the observed luminescence. ${ }^{28}$ Having most of the material removed from the substrate by ablation is also plausible explanation for the sharp drop in luminescence.

\section{CONCLUSION}

In conclusion, we have been able to obtain crystallized germanium nanoparticles in $\mathrm{SiO}_{2}$ matrix using femtosecond laser annealing. Laser pulses with pulse durations of 40, 80, 120 , and $160 \mathrm{fs}$ with various fluences were used for the irradiations. Raman spectra showed that we can fabricate germanium crystallites for fluencies higher than $45 \mathrm{~mJ} / \mathrm{cm}^{2}$. We identified that best crystallizations occur at $85 \mathrm{~mJ} / \mathrm{cm}^{2}$ with narrowest $\mathrm{Ge}$ phonon linewidth of $7.6 \mathrm{~cm}^{-1}$. Around $55 \mathrm{~mJ} / \mathrm{cm}^{2}$ longer pulse duration gives higher Raman intensity which may be due to dense crystallization at the longer pulse durations. For all pulse durations, swelling occur at the surface of the material for the fluencies higher than $40 \mathrm{~mJ} / \mathrm{cm}^{2}$ and ablation starts at $115 \mathrm{~mJ} / \mathrm{cm}^{2}$. For higher 
fluencies, defective crystalline material is left behind after ablation. Nonthermal microexplosions and nonlinear effects may play some role in femtosecond pulse laser crystallizations. Visible $\mathrm{PL}$ of $\mathrm{Si}_{0.2} \mathrm{O}_{0.5} \mathrm{Ge}_{0.3}$ thin films have been observed under the excitation radiation of $532 \mathrm{~nm}$ at room temperature. Broad PL peak around $600 \mathrm{~nm}$ was observed which is identified as defect center related luminescence. We have shown that femtosecond laser can be used to create crystallized material inside $\mathrm{SiO}_{2}$ matrices.

\section{ACKNOWLEDGMENTS}

The authors would like to thank Rasit Turan for XPS measurements. This work was supported by TUBITAK (The Scientific and Technological Research Council of Turkey) under Grant No. 109R037.

${ }^{1}$ Y. C. King, T. J. King, and C. Hu, IEEE Trans. Electron Devices 48, 696 (2001).

${ }^{2}$ W. K. Choi, W. K. Chim, C. L. Heng, L. W. Teo, V. Ho, V. Ng, D. A. Antoniadis, and E. A. Fitzgerald, Appl. Phys. Lett. 80, 2014 (2002).

${ }^{3}$ M. Zacharias and P. M. Fauchett, Appl. Phys. Lett. 71, 380 (1997).

${ }^{4}$ W. K. Choi, V. Ng, S. P. Ng, H. H. Thio, Z. X. Shen, and W. S. Li, J. Appl. Phys. 86, 1398 (1999).

${ }^{5}$ E. W. H. Kan, W. K. Chim, C. H. Lee, W. K. Choi, and T. H. Ng, Appl. Phys. Lett. 85, 2349 (2004).

${ }^{6}$ P. Punchaipetch, K. Ichikawa, Y. Uraoka, T. Fuyuki, A. Tomyo, E. Takahashi, and T. Hayashi, J. Vac. Sci. Technol. B 24, 1271 (2006).

${ }^{7}$ S. Tiwari, F. Rana, K. Chan, L. Shi, and H. Hanafi, Appl. Phys. Lett. 69, 1232 (1996)

${ }^{8}$ A. Kanjilal, J. Lundsgaard Hansen, P. Gaiduk, A. Nylandsted Larsen, N. Cherkashin, A. Claverie, P. Normand, E. Kapelanakis, D. Skarlatos, and D. Tsoukalas, Appl. Phys. Lett. 82, 1212 (2003).

${ }^{9}$ T. Takagahara and K. Takeda, Phys. Rev. B 46, 578 (1992).

${ }^{10}$ Y. Maeda, Phys. Rev. B 51, 1658 (1994).

${ }^{11}$ A. K. Dutta, Appl. Phys. Lett. 68, 1189 (1996).

${ }^{12}$ T. Shimizu-Iwayama, K. Fujita, S. Nakao, K. Saitoh, T. Fujita, and N. Itoh, J. Appl. Phys. 75, 7779 (1994).

${ }^{13}$ F. Gao, M. A. Green, G. Conibeer, E. Cho, Y. Huang, I. Wurfl, and C. Flynn, Nanotechnology 19, 455611 (2008).
${ }^{14}$ V. Cracium, C. B. Leborgne, E. J. Nicholls, and I. W. Boyd, Appl. Phys. Lett. 691506 (1996).

${ }^{15}$ M. Mulato, D. Toet, G. Aichmayr, and P. V. Santos, Appl. Phys. Lett. 70, 3570 (1997).

${ }^{16}$ J. Siegel, J. Solis, C. N. Afonso, and C. Garcia, J. Appl. Phys. 80, 6677 (1996).

${ }^{17}$ J. M. Shieh, Z. H. Chen, B. T. Dai, Y. C. Wang, A. Zaitsev, and C. L. Pan, Appl. Phys. Lett. 851232 (2004).

${ }^{18}$ O. Salihoglu, U. Kurum, G. Yaglioglu, A. Elmali, and A. Aydinli, J. Appl. Phys. 109, 123108 (2011).

${ }^{19}$ K. Sokolowski-Tinten, C. Blome, C. Dietrich, A. Tarasevitch, M. Horn von Hoegen, D. von der Linde, A. Cavalleri, J. Squier, and M. Kammler, Phys. Rev. Lett. 87, 225701 (2001).

${ }^{20}$ S. K. Sundaram and E. Mazur, Nature Mater. 1, 217 (2002).

${ }^{21}$ R. Skinner and R. E. Whitcher, J. Phys. E 5, 237 (1972).

${ }^{22}$ J. B. Lonzaga, S. M. Avanesyan, S. C. Langford, and J. T. Dickinson, J. Appl. Phys. 94, 4332 (2003).

${ }^{23}$ K. Piglmayer, E. Arenholz, C. Ortwein, N. Arnold, and D. Bauerle, Appl. Phys. Lett. 73, 847 (1998).

${ }^{24}$ E. N. Glezer and E. Mazur, Appl. Phys. Lett. 71, 882 (1997).

${ }^{25}$ A. Kaiser, B. Rethfeld, M. Vicanek, and G. Simon, Phys. Rev. B 61, 11437 (2000).

${ }^{26}$ Z. Guosheng, P. M. Fauchet, and A. E. Siegman, Phys. Rev. B 26, 5366 (1982).

${ }^{27}$ M. S. Rogers, C. P. Grigoropoulos, A. M. Minor, and S. S. Mao, Appl. Phys. Lett. 94, 011111 (2009).

${ }^{28}$ A. Dana, S. Tokay, and A. Aydinli, Mat. Sci. Semicond. Process 9, 848 (2006).

${ }^{29}$ M. Mulato, D. Toet, G. Aichmayr, P. V. Santos, and I. Chambouleyron, Appl. Phys. Lett. 70, 3570 (1997).

${ }^{30}$ H. Tang and I. P. Herman, Phys. Rev. B 43, 2299 (1991).

${ }^{31}$ A. Wellner, V. Paillard, C. Bonafos, H. Coffin, A. Claverie, B. Schmidt, and K. H. Heinig, J. Appl. Phys. 94, 5639 (2003).

${ }^{32}$ S. K. Gupta and P. K. Jha, Solid State Commun. 149, 1989 (2009).

${ }^{33}$ K. Kitahara, K. Ohnishi, Y. Katoh, R. Yamazaki, and T. Kurosawa, Jpn. J. Appl. Phys. 42, 6742 (2003).

${ }^{34}$ S.K. Ray and K. Das, Opt. Mater. 27, 948 (2005).

${ }^{35}$ W. K. Choi, Y. W. Ho, S. P. Ng, and v. Ng, J. Appl. Phys. 89, 2168 (2001).

${ }^{36}$ A. K. Dutta, Appl. Phys. Lett. 68, 1189 (1996).

${ }^{37}$ K. S. Min, K. V. Shcheglov, C. M. Yang, H. A. Atwater, M. L. Brongersma, and A. Polman, Appl. Phys. Lett. 68, 2511 (1996).

${ }^{38}$ S. T. Chang and S. H. Liao, J. Vac. Sci. Technol. B 27, 535 (2009). 